six weeks the surface was quite well covered with granulations.

September 15 we removed large Tiersch grafts from one thigh and covered the forehead and the right side of the head and dressed these with rubber tissue strips and dry gauze which were left on for one week. I carried one graft across an area of denuded bone to see if they would take on such a surface, as is asserted by some writers. The graft took finely at each end where it rested on granulation tissue, but died where it was in contact with denuded bone. The grafts took well, but the patient rolled her head on the pillow and crumpled some of the grafts on the side of the head, thus losing some of the surface that was covered at the first sitting; but the grafts lived.

September 28, we grafted the remainder of the head, dressed it as before, and kapt the patient sitting up for fourteen days to prevent rubbing off the grafts. She sat in a lowbacked chair, and when she slept she leaned her head on her arm, which rested on a table in front of her. As the grafts on the forehead were well taken, at the end of five days the dressings were changed and redressed with rubber strips, and normal salt solution was used to keep the dressings moist for five days more, after which sterile petrolatum was spread on gauze and applied to the grafted surface.

The latter dressing seemed ideal, as the grafts flourished and the surfaze cleared up with great rapidity, so that at the end of two months from the day of the injury, the patient returned home with a new scalp and in fair health.

Figure 1 was taken two weeks after the grafts were applied and shows some denuded spots still bare of grafts. Figure 2 was taken on the day the patient left for her home, just two months from the day of her injury.

\section{EXTENSIVE DESTRUCTION OF VULVA AND ADJACENT TISSUES PROBABLY DUE TO PNEUMOCOCCIC INFECTION}

Guthbert Powell, M.D., Denver

Fellow of the American College of Surgeons

On October 5, last, a white woman, aged 48 , was admitted to my service at the Denver City and County Hospital. Her history, briefly, is as follows:

Patient was married at 16 and has been a widow for twenty-seven years. She has never been pregnant. Menstruation every thirty days, a normal four-day flow. Her past health has been good; has had no illness of any kind during the past fifteen years. Family history not noteworthy.

Her present trouble began two weeks before admission to the hospital, when a vulva pad worn during her menstrual flow chafed and irritated the parts. A week ago she procured some medicine from a physician to be used locally as a wash. She is positive that the wash did not smell of carbolic acid. Two days before admission to the hospital, the labia become greatly swollen, painful and dark colored.

The patient on admission was thin, pale and emaciated. Temperature 100.2, pulse about 110 , bowels constipated, appetite and digestion good. Urine contained a trace of albumin, a few hyaline and granular casts, some pus cells; sugar was absent. A Wassermann test was negative. There was no glandular involvement.

External examination of the genitals revealed an extensive foul smelling ulceration, partly covered with a black necrotic mass. The area involved extended from above the pubic promontory to below the anus, and, laterally, well outside of the labia majora on to the inner surface of the thighs.

Under ether anesthesia, an examination of the pelvic organs was made per vaginam. The bladder was found greatly distended, rising as high as the umbilicus and was emptied by catheter. The uterus, tubes and ovaries were apparently normal, as was also the vagina.

The necrotic mass was snipped off with scissors, showing an extensive destruction of the underlying fat and connective tissue, leaving the perineal muscles and lower 2 inches of rectum exposed as shown in the illustration.
Pure carbolic acid was swabbed over the raw surfaces followed immediately by alcohol, and a dusting powder of equal parts iodoform and boric acid was applied.

The laboratory report of cultures made from the necrotic mass and smears taken from the. raw surface showed large numbers of pneumococci present, with a few streptococci. No spirilla were demonstrated.

The subsequent treatment of the case consisted of cleansing once daily, with a weak solution of hydrogen peroxid and the application of the iodoform and boric acid powder. The patient was kept in bed. The temperature, after the removal of the slough, dropped to normal, where it remained during convalescence. It was necessary to catheterize the patient every eight hours until October 16, after which date urine was voided normally.

Healing progressed steadily; at the end of the fourth week, almost the whole of the ulcerated area had filled in and healed over. There was a surprisingly small amount of scar tissue visible, the normal skin seemingly having covered the greater part of the denuded area, growing in from the edges.

The patient left the hospital November 10, the thirty-sixth day after her admission, entirely healed with the exception of a very narrow margin of granulation tissue about the

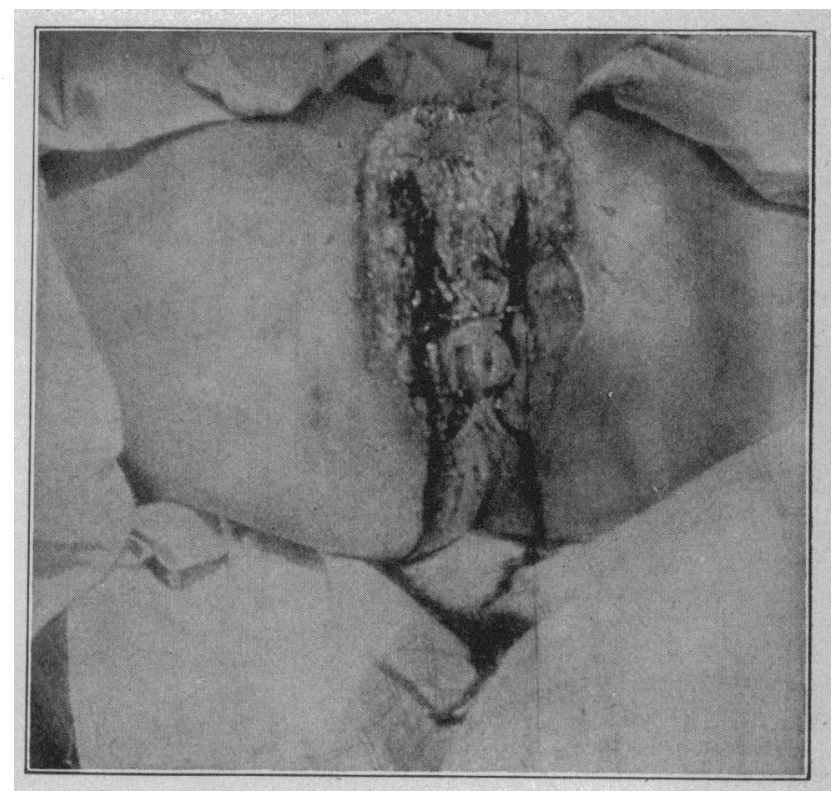

Uicerated area showing extensive destruction of superficial tissues witl exposure of periaeal muscles and lower two inches of rectum.

vaginal opening. The parts presented a remarkably smooth and normal appearance in spite of the extensive destruction of tissue which had taken place. Her general condition also was greatly improved; weight had increased and color was good. Unfortunately she left the hospital before a photograph showing the healing of the parts was taken.

Dr. A. J. Markley of Denver has a photograph of a patient with identically the same lesions as shown in the illustration. This patient was seen by him in February, 1912, and his account of her condition coincides exactly with my case. Bacteriologic examinations of Dr. Markley's patient showed a pure culture of pneumococcus. No other growth was found; she made a complete recovery in about thirty days.

There are several interesting points to be noted in this case.

1. Its rarity; a brief search of the literature fails to find mention of a similar case.

2. The bacteriologic findings; pneumococci in large numbers, with a comparative absence of other organisms.

3 . The rapidity of the destructive process.

4. The readiness with which healing took place, and the remarkably good cosmetic appearance, in spite of the extensive tissue destruction.

432 Metropolitan Building. 